\title{
Comunicación de la responsabilidad social empresarial de las empresas de América Latina en Facebook: estudio comparativo con las empresas globales
}

\section{Paul Capriotti ${ }^{1}$ Ileana Zeler ${ }^{2}$}

Recibido: 01/05/2018

Aprobado por pares: 23/10/2018
Enviado a pares: 22/05/2018

Aceptado: 08/01/2019

DOI: $10.5294 /$ pacla.2020.23.2.7

Para citar este artículo / to reference this article / para citar este artigo Capriotti, P. y Zeler, I. (2020). Comunicación de la responsabilidad social empresarial de las empresas de América Latina en Facebook: estudio comparativo con las empresas globales. Palabra Clave, 23(2), e2327. https://doi.org/10.5294/pacla.2020.23.2.7

\section{Resumen}

El nuevo contexto organizacional en América Latina cambió la manera en que las empresas se relacionan con sus públicos. La responsabilidad social empresarial (RSE) puede ayudar a las empresas latinoamericanas a lograr mayor integración en el entorno social y fortalecer las relaciones con sus públicos. Las redes sociales, particularmente Facebook, pueden ser herramientas claves para la comunicación de las actividades de RSE, ya que contribuyen positivamente a la reputación corporativa. El objetivo de este estudio es analizar la comunicación de la RSE de las principales empresas de América Latina en Facebook. La investigación incluye un análisis comparativo con las principales empresas globales y revela que las empresas tratan los temas sociales con menos frecuencia que los temas económicos en Facebook. Las empresas usan Facebook como un canal de difusión más

\footnotetext{
https://orcid.org/0000-0002-9398-5886. Universitat Rovira i Virgili, España. paul.capriotti@urv.cat

$2 \bowtie$ https://orcid.org/0000-0002-5550-1000. Universitat Rovira i Virgili, España. ileana.zeler@urv.cat
} 
que como un canal de comunicación de su RSE, por lo que se hace necesario un enfoque más interactivo y dialógico en esta red social.

\section{Palabras clave (Fuente: tesauro de la Unesco)}

Internet; redes sociales; comunicación; responsabilidad social; empresas. 


\section{Disseminating Latin American Companies' Corporate Social Responsibility on Facebook: A Comparative Study with Global Companies}

\section{Abstract}

The new Latin American organizational context changed the way companies relate to audiences. Corporate social responsibility (CSR) can help Latin American companies to better integrate into their social environment and strengthen relations with their audiences. Social media, particularly Facebook, can be key tools for disseminating CSR endeavors, as they positively contribute to corporate reputation. This study intends to analyze CSR communication on Facebook by major Latin American companies. The research includes a comparative analysis of these companies and reveals that they deal with social issues less often than economic issues on Facebook. Companies use Facebook as a broadcasting medium rather than as a communication channel for their CSR, so a more interactive and dialogic approach is needed for this social media website.

\section{Keywords (Source: Unesco Thesaurus)}

Internet; social media; communication; social responsibility; business. 


\section{Comunicação da responsabilidade social empresarial das empresas da América Latina no Facebook: estudo comparativo com as empresas globais}

\section{Resumo}

O novo contexto organizacional na América Latina mudou a forma como as empresas se relacionam com seu público-alvo. A responsabilidade social empresarial (RSE) pode ajudar as empresas latino-americanas a conseguir mais integração no contexto social e fortalecer as relações com seus públicos. As redes sociais, em especial o Facebook, podem ser ferramentas-chave para comunicar as atividades de RSE, já que contribuem de forma positiva para a reputação corporativa. O objetivo deste estudo é analisar a comunicação da RSE das principais empresas da América Latina no Facebook. Esta pesquisa inclui uma análise corporativa com as principais empresas globais e revela que as empresas tratam os temas sociais com menos frequência que os temas econômicos no Facebook. As empresas o usam como canal de difusão mais do que como canal de comunicação de sua RSE, por isso é necessária uma abordagem mais interativa e dialógica nessa rede social.

\section{Palavras-chave (Fonte: tesauro da Unesco)}

Internet; redes sociais; comunicação; responsabilidade social; empresas. 


\section{Introducción}

América Latina ha experimentado un crecimiento significativo económico en los últimos veinte años. El ingreso de capital extranjero a la región generó la expansión y el crecimiento de las empresas, así como el surgimiento de nuevas grandes empresas (Alburquerque, 2004; Yudira, 2007). Este nuevo contexto organizacional generó la necesidad de aumentar la confianza y la transparencia, no solo desde el punto de vista de los beneficios económicos, sino también de la responsabilidad social. La necesidad de adquirir mayor competitividad y de responder a las responsabilidades económicas y sociales permite integrar los aspectos sociales y medioambientales en la estrategia empresarial y llevar a cabo prácticas más responsables, particularmente en cuestiones laborales y sociales que son relevantes en el contexto regional (Peinado-Vara, 2011). La responsabilidad social empresarial (RSE) puede ayudar a las empresas latinoamericanas a lograr la integración en el entorno social para satisfacer $y$ fortalecer las relaciones, y lograr una mayor aceptación y reconocimiento por parte de sus públicos.

La comunicación de la RSE se ha vuelto un aspecto importante para mejorar la relación entre las empresas latinoamericanas y sus públicos. Las redes sociales están cambiando la comunicación de la RSE, se han convertido en una gran herramienta de comunicación one-to-one para las empresas y han modificado la manera en que se relacionan con sus públicos. Las redes sociales permiten a las empresas comunicar sus actividades de RSE y establecer relaciones interactivas con sus públicos (Taylor \& Kent, 2014). Algunos estudios muestran que la participación activa de las empresas en las redes sociales está asociada positivamente a la reputación corporativa (Dijkmans, Kerkhof \& Beukeboom, 2015), por lo que las empresas reconocen el potencial de las redes sociales y las están integrando cada vez más a sus programas de comunicación.

Facebook, la red social con mayor penetración y popularidad, ofrece la posibilidad de crear y difundir contenido de RSE, así como de dialogar y escuchar activamente a los públicos de las organizaciones. La gran aceptación de Facebook por parte de los usuarios y su crecimiento exponencial en los últimos años en los principales países de América Latina (Fosk, 
2015) favoreció la incorporación de estas redes sociales a los programas de comunicación de las empresas. Así, la comunicación a través de Facebook en el contexto latinoamericano se convierte en un instrumento clave de la comunicación de RSE en las empresas para aumentar su visibilidad e impulsar la interacción, el diálogo y el compromiso con sus públicos (Moreno et al., 2017).

Comparar las empresas de América Latina con las empresas globales permite conocer cómo estas están posicionadas en el contexto global y analizar si están llevando adelante una mejor o peor gestión de la comunicación de la RSE en Facebook en relación con las empresas globales.

Por tanto, el objetivo general de esta investigación es analizar la comunicación de la RSE de las empresas de América Latina en Facebook. A partir de este objetivo general, se plantearon los siguientes objetivos específicos: a) identificar si las empresas usan Facebook para comunicar sus actividades de RSE y evaluar qué nivel de actividad tienen en la red social, b) analizar los contenidos de RSE que las empresas difunden en la red social y c) estudiar los recursos de información utilizados por las empresas para difundir los contenidos de RSE en la red social.

\section{La comunicación de la RSE en el entorno digital}

Internet y las redes sociales han contribuido a la evolución de la comunicación de la RSE de las empresas y han pasando de una perspectiva comunicativa tradicional y unidireccional a una perspectiva comunicacional más interactiva y bidireccional. Las plataformas digitales han transformado la forma en la que los usuarios obtienen información, se comunican y se relacionan con las organizaciones (Guillory \& Sundar, 2014; Ure, 2016). Las organizaciones encuentran en internet y las redes sociales canales para difundir información y dialogar sobre sus responsabilidades sociales y medioambientales (Capriotti \& Moreno, 2007).

Este nuevo contexto comunicativo organizacional está cambiando la forma en la que se entiende la comunicación de la RSE y los modelos que han inspirado su comunicación hasta ahora. Hace veinte años, Esrock \& 
Leichty (1998) señalaron que las empresas estaban utilizando su presencia en internet para mejorar su reputación, a través de la comunicación de sus actividades de RSE. Autores más recientes como Kim (2017) agregan que la comunicación de los atributos sociales puede tener un impacto positivo en la reputación de las organizaciones. Y algunos estudios indican que mayoritariamente los profesionales de la comunicación consideran que la comunicación de la RSE es muy importante y debe ser informada a los públicos (Navarro, Moreno \& Al-Sumait, 2017). Sin embargo, los efectos positivos de la reputación se producen cuando los públicos participan en el proceso de comunicación y la formulación de estas actividades. Por esta razón, promover el diálogo con los públicos a través del proceso de comunicación es necesario para fortalecer la reputación corporativa.

Las redes sociales generan un ecosistema de comunicación en línea óptimo para la comunicación interactiva de las organizaciones con sus públicos. Varios autores afirman que las redes sociales son herramientas clave para la comunicación de la RSE (Capriotti, 2017; Cortado \& Chalmeta, 2016). Y la investigación demuestra que la participación activa de las empresas en las redes sociales puede tener un impacto positivo en la reputación corporativa (Dijkmans, Kerkhof \& Beukeboom, 2015).

Entre las redes sociales disponibles, Facebook es la red social con mayor número de usuarios activos a nivel mundial (Kemp, 2017) y mayor crecimiento en América Latina (Fosk, 2015). Además de aumentar la visibilidad, escuchar activamente y generar interacción con los públicos, Facebook permite a las empresas crear perfiles relacionados con diferentes áreas de la organización. Sin embargo, el hecho de crear perfiles en Facebook no significa una alta participación y visibilidad. Para que esto suceda, es necesario entender las redes sociales como una herramienta de diálogo e interacción con los públicos (Waters, Burnett, Lamm \& Lucas, 2009).

Las empresas tienen una presencia mayoritaria en Facebook (Ki \& Nekmat, 2014), pero su nivel de actividad en la red social por lo general es baja. Los diferentes estudios muestran que las empresas publican menos de una publicación por día (Devaney, 2015; Quintly, 2016) o al menos una 
publicación por día (Estudio de Comunicación, 2017). Sin embargo, los expertos y profesionales indican que se debería publicar entre 1 y 2 posts por día para tener una frecuencia adecuada en Facebook (Jordan, 2017; Patel, 2016). Por tanto, un primer objetivo específico es analizar el nivel de presencia y actividad que las empresas de América Latina mantienen en esta red social.

\section{La comunicación de los temas económicos y sociales en el entorno digital}

El concepto de RSE ha ido evolucionando gradualmente desde la década de 1950. A lo largo del tiempo, su definición ha estado vinculada con las responsabilidades del "hombre de negocios" (Carroll, 1999), con las responsabilidades sociales de las empresas y con la ciudadanía corporativa (Waddock, 2004). Varios autores que analizan la evolución de la RSE (Carroll, 1999, 2016; Masoud, 2017; Waddock, 2004; Wood, 1991) concuerdan que en evolución gradual de la definición de la RSE coexisten diversos conceptos.

Las empresas tienen dos responsabilidades corporativas básicas: las responsabilidades económico-legales y las responsabilidades socio-ético-filantrópicas de una empresa (Carroll, 1999, 2016; Masoud, 2017; Waddock, 2004; Wood, 1991). El rol económico se relaciona con las responsabilidades de las organizaciones para elaborar productos y brindar servicios de calidad, cumplir con la ley y obtener beneficios. El rol social se relaciona con las responsabilidades de las organizaciones en pos de respetar los derechos humanos y laborales, cuidar el medio ambiente y hacer un aporte social a la comunidad (Capriotti \& Pardo, 2012).

La manera en que estos roles se comunican a los públicos puede influir en las asociaciones mentales que las personas tienen sobre las empresas, es decir, en la reputación corporativa. Sin embargo, las actividades de RSE se suelen comunicar con escasa frecuencia en las redes sociales. Las diferentes investigaciones demuestran que las empresas tienden a centrar su comunicación en los temas económicos en lugar de los temas sociales (Cho, Furey \& Mohr, 2016; Haigh, Brubaker \& Whiteside, 2013). Por tan- 
to, un segundo objetivo específico de este estudio es conocer qué tipo de contenido de RSE están difundiendo las empresas en Facebook.

Para lograr una comunicación efectiva con los públicos, Facebook ha puesto a disposición de las empresas diferentes recursos de información. Estos recursos les permiten crear y difundir contenidos integrando diferentes recursos gráficos (texto, imagen y emoticón), recursos interactivos (link, etiqueta a usuarios y hashtag) y recursos audiovisuales (video-audio e imagen animada).

Los estudios indican que los recursos de información ayudan a las organizaciones a mejorar el alcance y la interacción en Facebook (Invodo, 2016; Von Leoprechting, 2016) y aumentar el compromiso si crean mensajes que alienten el diálogo con sus públicos (Abitbol \& Lee, 2017). Las organizaciones están creando espacios en las redes sociales para compartir información relacionada con las actividades de RSE, establecer una conexión con los usuarios e invitarlos a interactuar con las empresas. Pero algunas investigaciones indican que las organizaciones tienden a usar más los recursos gráficos que los recursos audiovisuales e interactivos (Luarn, Lin \& Chiu, 2015). Por tanto, un tercer objetivo específico para esta investigación es conocer qué tipo de recursos de información utilizan las empresas para difundir su contenido de RSE en Facebook.

\section{Metodología}

Para esta investigación, se seleccionaron las empresas con mejor reputación corporativa, por ser consideradas referentes en la gestión de la comunicación estratégica, a la vez que son las que destinan importantes recursos para innovar en comunicación digital y son líderes en gestión de RSE.

Para elegir las empresas de América Latina, se tomaron como referencia los países con mayor PIB presentes en el Anuario Estadístico de América Latina y el Caribe de la Comisión Económica para América Latina y el Caribe (Cepal), y en los cuales se haya realizado el estudio anual de reputación corporativa Merco Empresas del Monitor Empresarial de Reputación Corporativa (Merco), al menos durante dos años. Así, se seleccionaron las 
35 primeras empresas presentes en los rankings de Brasil, México, Argentina, Colombia, Chile y Perú.

Para evaluar comparativamente las empresas de América Latina con las empresas globales, se tomó como referencia el ranking anual de reputación corporativa Global RepTrak ${ }^{\circledast} 100$ del Reputation Institute, y se seleccionaron las 35 primeras empresas presentes en el estudio al menos dos años.

Los perfiles de Facebook fueron seleccionados a partir de las empresas que cuentan con fanpages corporativas a nivel nacional (en el caso de América Latina) e internacional (en el caso de las empresas globales) en 2015 y 2016. Así, la muestra final incluyó 135 perfiles de Facebook de empresas que operan en América Latina (25 de Brasil, 19 de México, 23 de Argentina, 27 de Colombia, 19 de Chile y 22 de Perú), y 24 empresas que operan a nivel mundial.

El volumen total de posts identificados durante 2015 y 2016 fue de 137 841. Para seleccionar la muestra de los posts, se eligieron dos semanas por mes, durante 12 meses. Se recopilaron los posts de las semanas impares de enero a junio de 2015 y las semanas pares de julio a diciembre de 2016. Finalmente, se obtuvieron 32760 posts.

Para alcanzar los objetivos establecidos, se definieron tres preguntas de investigación (RQ):

- $\quad$ RQ1: ¿Están las empresas utilizando Facebook para compartir información sobre sus actividades de RSE? ¿Qúe nivel de actividad tienen las empresas en la red social?

- RQ2: ¿Qué tipo de contenidos de RSE están difundiendo las empresas en Facebook?

- RQ3: ¿Qué tipo de recursos de información están usando las empresas para difundir los contenidos de RSE en Facebook?

Para responder a las preguntas de investigación, se definieron tres categorías basadas en la presencia y la actividad (RQ1), el contenido (RQ2) y 
los recursos comunicativos (RQ3). Estas categorías fueron desarrolladas y testeadas en investigaciones previas (Capriotti, Carretón \& Castillo, 2016; Losada-Díaz \& Capriotti, 2015).

Para la RQ1, se estableció la categoría de presencia y actividad. Se buscó identificar los perfiles desarrollados por las empresas en Facebook y evaluar el volumen de contenidos difundidos por estas. Para ello, se determinaron tres dimensiones de análisis (dos de presencia y una de actividad), el tipo de página corporativa (fanpage corporativa o perfil de usuario), otras páginas específicas (si la empresa presenta otros perfiles comerciales, de fundaciones, de RSE o de proyectos de RSE) y el nivel de actividad (obtenido a partir del promedio diario y semanal de las publicaciones de las empresas).

Para la RQ2, se estableció la categoría de contenido, la cual permite identificar los principales temas tratados por las empresas. Para ello, se identificaron tres dimensiones claves:

1. Temas de negocios: temas relacionados con las actividades empresariales, comerciales y de negocios de las empresas. Para esta dimensión, se identificaron cuatro tópicos:

a. Las estrategias (información vinculada con las políticas y estrategias empresariales).

b. Los resultados (información sobre resultados de negocios, financieros, económicos y bursátiles.

c. La oferta (información relacionada con las características de los productos, los servicios y las marcas).

d. La innovación (información sobre los aspectos claves de la innovación creativa y productiva.

2. Temas de RSE: temas relacionados con las actividades de RSE de la empresa en sus actividades cotidianas. Para esta dimensión, se determinaron tres tópicos:

a. La integridad (información vinculada a los compromisos, estrategias, políticas y prácticas responsables en dirección de la empresa y de la gestión comercial y de marketing). 
b. El trabajo (información sobre aspectos laborales y de empleo de la empresa e información sobre los compromisos, las estrategias, las políticas y las prácticas responsables en la gestión de los recursos humanos de la organización).

c. La ciudadanía (información relacionada con los compromisos, las estrategias, las políticas, las prácticas responsables de la empresa a nivel social y medioambiental).

3. Temas de contexto: temas relacionados con la situación general y del sector empresarial de la empresa. Para esta dimensión, se señalaron tres tópicos:

a. El contexto general (aspectos que no tienen que ver con la actividad del negocio).

b. El contexto sectorial (aspectos que tienen que ver con la actividad del negocio).

c. El contexto relacional (aspectos que tienen que ver con la relación de la empresa con los usuarios).

Para la RQ3, se estableció la categoría recursos comunicativos, que permite determinar los recursos utilizados para la difusión de los contenidos. Así, se definieron los siguientes recursos: gráficos (texto, imagen o emoticón), interactivos (link, hashtag o etiqueta a usuarios) y audiovisuales (imagen animada o audio-video). En este análisis, puede incluirse más de un recurso posible.

La metodología utilizada fue el análisis de contenido de posts. Para la recolección de los datos, se utilizó una herramienta de monitorización disponible en internet llamada Fanpage Karma. El planteamiento de las categorías de análisis permitió el diseño de una plantilla de análisis basada en Excel, en la cual se volcaron y codificaron los datos recopilados de la muestra (anexo 1).

\section{Resultados}

Los resultados fueron organizados de acuerdo con las preguntas de investigación formuladas para este estudio (RQ). 


\section{Presencia y actividad}

En cuanto a la categoría presencia y actividad (RQ1), los resultados indican que mayoritariamente las empresas cuentan con fanpages corporativas y tienen una destacada presencia de páginas de Facebook vinculadas a las actividades de RSE. Asimismo, su actividad es bastante frecuente en la red social.

La presencia en Facebook de las empresas de América Latina es de alrededor del $85 \%$ (tabla 1). Además de fanpages corporativas, las empresas tienen una notable presencia de fanpages relacionadas con la RSE (RSE, proyectos de RSE y fundación), que alcanza alrededor del $30 \%$. Esto significa que un tercio de las empresas están usando la red social para dar a conocer proyectos sociales, así como su compromiso con la comunidad y el medio ambiente. Sin embargo, cabe señalar que hay más fanpages comerciales que de RSE y están presentes en más del $50 \%$ de las empresas.

\section{Tabla 1. Presencia de las empresas en Facebook}

\begin{tabular}{|c|c|c|}
\hline & Latinoamericanas & Globales \\
\hline & \multicolumn{3}{|c|}{ Fanpages corporativas (en \%) } \\
\hline Sí & 86,5 & 75,0 \\
\hline No & 13,5 & 25,0 \\
\hline Perfiles RSE & \multicolumn{3}{|c|}{ Otras páginas específicas (\%) } \\
\hline Perfiles comerciales & 29,6 & 37,5 \\
\hline
\end{tabular}

Fuente: elaboración propia.

Al comparar la presencia de las empresas de América Latina y las empresas globales, se encuentra que las empresas globales también tienen una alta presencia en Facebook (75 \%). Esta presencia es menor que la que tienen las empresas de América Latina en la red social, que superan en más de un $10 \%$ a las empresas globales. En cuanto a las páginas específicas encontradas, se detecta que las empresas globales tienen mayor presencia de perfiles de RSE y comerciales en la red social que las empresas 
de América Latina. Si bien la diferencia es poco significativa en relación con los perfiles de RSE (menos del $10 \%$ más que las empresas de América Latina), se detecta una diferencia bastante significativa en los perfiles comerciales. Casi el $80 \%$ de las empresas globales cuentan con perfiles vinculados a productos, servicios o marcas, un $23 \%$ más que las empresas de América Latina.

En relación con la actividad, las empresas publican contenidos con bastante frecuencia en sus fanpages corporativas. Sin embargo, las empresas de América Latina publican contenidos con mayor frecuencia que las empresas globales. Durante el análisis se encontró que cada empresa de América Latina publicó en promedio casi 1,2 posts por día. Esto significa entre uno y dos posts más por semana que las empresas globales, las cuales publican menos de un post por día (tabla 2).

\section{Tabla 2. La actividad de las empresas en Facebook}

\begin{tabular}{|c|c|c|}
\hline & Latinoamericanas & Globales \\
\hline Promedio/empresa/día & 1,2 & 0,9 \\
\hline Promedio/empresa/semana & 8,4 & 6.0 \\
\hline
\end{tabular}

Fuente: elaboración propia.

En cuanto a la actividad por temas tratados por las empresas en Facebook (tabla 3), se puede observar que los temas de RSE se encuentran muy por debajo de la media de actividad sugerida. Los posts analizados representan una media de 0,2 posts por día por empresa de América Latina. A diferencia de ello, los posts relacionados con los temas comerciales representan 3 veces más que la media de posts de RSE por día por empresa $(0,7$ por día por empresa), y se aproximan mucho más a la media de actividad sugerida. Las empresas globales presentan resultados similares a las empresas de América Latina. Los posts de negocios representan 7 veces más que los posts de RSE, y se aproximan de manera notable a la media de publicaciones sugerida. 


\section{Tabla 3. Actividad de negocios, RSE y contexto de las empresas en Facebook}

\begin{tabular}{|c|c|c|c|c|}
\hline & & Negocios & RSE & Contexto \\
\hline \multirow{3}{*}{ Latinoamericanas } & Promedio/día & 0,7 & 0,2 & 0,3 \\
\cline { 2 - 5 } & Promedio/semana & 4,8 & 1,6 & 1,9 \\
\hline \multirow{2}{*}{ Globales } & Promedio/día & 0,7 & 0,1 & 0,1 \\
\cline { 2 - 5 } & Promedio/semana & 4,7 & 0,5 & 0,8 \\
\hline
\end{tabular}

Fuente: elaboración propia.

\section{Contenido}

En relación con la categoría contenido (RQ2), las empresas se centran más en la difusión de contenidos referidos a los temas de negocios que en la difusión de temas de RSE. También divulgan bastante información sobre temas de contexto (tabla 4).

\section{Tabla 4. Contenido de las empresas en Facebook (\%)}

\begin{tabular}{|c|c|c|c|c|c|c|c|c|c|c|}
\hline \multicolumn{6}{|c|}{$\operatorname{Negocios}(\%)$} & \multicolumn{4}{|c|}{ RSE (\%) } & \multirow{2}{*}{$\begin{array}{c}\begin{array}{c}\text { Contexto } \\
(\%)\end{array} \\
\text { 氶 }\end{array}$} \\
\hline & 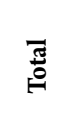 & 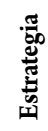 & 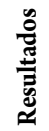 & & 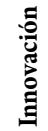 & 䒕 & 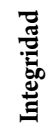 & 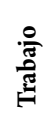 & 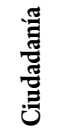 & \\
\hline Latinoamericanas & 57,8 & 12,6 & 1,5 & 41,2 & 2,5 & 19,6 & 2,8 & 4 & 12,9 & 22,6 \\
\hline Global & 77,1 & 18,8 & 0,4 & 49,8 & 8,1 & 9,0 & 1,4 & 2,6 & 5,0 & 13,9 \\
\hline
\end{tabular}

Fuente: elaboración propia.

La mayoría de las publicaciones de las empresas de América Latina están relacionadas con la oferta de productos y servicios (negocios) y, en menor medida, con la ciudadanía (RSE) y la estrategia (negocios). Los otros temas, como los resultados, la innovación, la integridad y el trabajo, están por debajo del $4 \%$, con muy poca relevancia. Los resultados muestran que los temas de negocios alcanzan casi el $60 \%$ de la muestra de posts $(57,8 \%)$, mientras que los temas de RSE son menos del $20 \%$. Las empresas de América Latina publican similar cantidad de posts de RSE y de contexto (alrededor del $20 \%$ ). 
Existen diferencias significativas con las empresas globales. Cabe destacar que las empresas globales publican más contenidos de negocios $(77,1$ $\%)$, y menos contenidos de RSE (9\%) y de contexto (14\%) que las empresas de América Latina. Al igual que las empresas latinoamericanas, las globales difunden más publicaciones de contexto que de RSE (se encontró casi un $15 \%$ de posts de contexto y menos de un $10 \%$ de posts de RSE).

Del total de publicaciones relacionadas sobre los temas de negocios (tabla 5), casi tres cuartas partes de los posts de las empresas de América Latina corresponden a la oferta comercial $(71,3 \%)$. En un porcentaje bastante menor, se detectan publicaciones sobre temas relacionados con la estrategia empresarial (alrededor del $20 \%$ ), y en muy pocos casos se encuentran posts sobre la innovación productiva $(4,3 \%)$ y los resultados de negocios, financieros, económicos y bursátiles $(2,6 \%)$.

Los resultados de las empresas globales muestran diferencias algo significativas con los de las empresas de América Latina. Las empresas globales mayoritariamente comparten temas relacionados con la oferta $(64,6 \%)$. Además, también un porcentaje significativo de posts sobre temas de la estrategia empresarial (casi el $25 \%$ ). Se debe destacar que las empresas globales difunden bastante más temas de innovación (10\%). A pesar de que este porcentaje es bajo, supera más de 2 veces el porcentaje de posts sobre innovación de las empresas de América Latina. Asimismo, prácticamente las empresas mundiales no publican contenidos sobre resultados (tabla 5).

\section{Tabla 5. Temas de negocios tratados por las empresas (\%)}

\begin{tabular}{|c|c|c|c|c|}
\hline & Estrategia & Resultados & Oferta & Innovación \\
\hline Latinoamericanas & 21,8 & 2,6 & 71,3 & 4,3 \\
\hline Globales & 24,3 & 0,6 & 64,6 & 10,5 \\
\hline
\end{tabular}

Fuente: elaboración propia

En cuanto a los temas de RSE, en la tabla 6 se puede ver que más de dos tercios de los posts de las empresas de América Latina están relacionados con la ciudadanía (65,6\%), sobre aspectos sociales y medioambien- 
tales de las empresas. Los temas de integridad (14,2\%) y de trabajo (20,2\%) tienen una presencia 3 veces menor que los primeros.

En relación con las empresas globales, se observa que hay algunas diferencias significativas. En la tabla 6, se puede ver que las empresas globales también difunden principalmente contenidos de ciudadanía, aunque un $10 \%$ menos que las empresas de América Latina (alrededor del $55 \%$ ). Asimismo, otra diferencia es que las empresas globales les dan mayor tratamiento a los temas de trabajo que las empresas de América Latina (en casi el $30 \%$ de los posts). En cuanto a los temas de integridad, los resultados de las empresas globales son similares a los de las empresas de América Latina (alrededor del $15 \%)$.

\section{Tabla 6. Temas de RSE tratados por las empresas (en \%)}

\begin{tabular}{|c|c|c|c|}
\hline & Integridad (\%) & Trabajo (\%) & Ciudadanía (\%) \\
\hline Latinoamericanas & 14,2 & 20,2 & 65,6 \\
\hline Global & 15,7 & 28,9 & 55,4 \\
\hline
\end{tabular}

Fuente: elaboración propia

\section{Recursos comunicativos}

En cuanto a los recursos comunicativos (RQ3), los resultados muestran que los recursos gráficos (texto e imágenes) son los más utilizados por las empresas para difundir los contenidos de RSE en Facebook (tabla 7).

Casi la totalidad de los posts de las empresas de América Latina tienen texto $(97,8 \%)$ y mayoritariamente están acompañados de una o más imágenes $(77,3 \%)$. En pocos casos, se incluyen los emoticones en las publicaciones. Si bien este recurso fue introducido por Facebook a lo largo del tiempo, se ha encontrado que algo más del $10 \%$ de las publicaciones cuentan con este recurso gráfico.

Además, las empresas utilizan recursos interactivos tales como links (40\%) y hashtags (50\%), aunque en bastante menor porcentaje que el texto y la imagen. En pocos casos, los posts también cuentan con etiquetas a 
otros usuarios (15\%). Esto sugiere que un grupo amplio de empresas están ya implementando estos recursos para fomentar la interacción con los usuarios en Facebook.

Los recursos audiovisuales son los menos utilizados por las empresas de América Latina en Facebook. Los resultados señalan que algo más del $10 \%$ de los posts contienen audio/videos (12,7\%). También se ha detectado que casi un $10 \%$ de los posts cuentan con imágenes animadas.

Estos resultados no muestran una diferencia muy significativa con los resultados de las empresas globales. Sin embargo, cabe destacar que las empresas globales usan más frecuentemente los recursos audiovisuales en sus posts que las empresas de América Latina en su conjunto. Si bien el porcentaje es del $26 \%$, esto supone 2 veces el porcentaje de las empresas de América Latina. También, las empresas globales prácticamente no incluyen emoticones en sus posts (4\%), a diferencia de las empresas de América Latina que lo suelen utilizar, aunque en pocas ocasiones $(12,1 \%)$.

\section{Tabla 7. Recursos de información de las empresas en Facebook (\%)}

\begin{tabular}{|c|c|c|c|c|c|c|c|c|}
\hline & \multicolumn{3}{|c|}{ Gráficos (\%) } & \multicolumn{2}{c|}{ Interactivos (\%) } & \multicolumn{2}{c|}{ Audiovisuales (\%) } \\
\cline { 2 - 9 } & Texto & Imagen & Emoticón & Etiqueta & Hashtag & Link & $\begin{array}{c}\text { Imagen } \\
\text { animada }\end{array}$ & Audio/video \\
\hline Latinoamericanas & 97,8 & 77,3 & 12,1 & 15,8 & 40,6 & 48,6 & 8,6 & 12,7 \\
\hline Global & 97,2 & 69,7 & 4,0 & 24,2 & 46,3 & 53,7 & 0,0 & 26,0 \\
\hline
\end{tabular}

Fuente: elaboración propia.

\section{Discusión y conclusiones}

A pesar de que tanto las empresas de América Latina como las empresas globales tienen amplia presencia en Facebook, en los resultados se detectó que un gran número de empresas todavía no incorporan esta red social en sus estrategias de comunicación (15\% en las empresas de América Latina y $25 \%$ en las empresas globales). Asimismo, las empresas de América Latina que están presentes tienen un buen nivel de actividad en Facebook. 
Según la recomendación de expertos y profesionales, la media adecuada sería de 1 a 2 posts al día (Jordan, 2017; Patel, 2016). Los resultados obtenidos muestran que las empresas están publicando con bastante frecuencia, aunque cabe destacar que las de América Latina publican con mayor frecuencia que las empresas globales. Estos resultados son similares a los de otros estudios realizados (Estudio de Comunicación, 2017; Kim, Kim \& Hoon Sung, 2014).

Las compañías se están sumando a la tendencia del uso de Facebook para la gestión de la comunicación online de la RSE (Cortado \& Chalmeta, 2016), pero aún le dan mayor espacio a los aspectos comerciales. Las empresas están utilizando Facebook para crear y gestionar fanpages relacionadas con las áreas organizativas, principalmente lo comercial. Estos resultados se encuentran en línea con estudios previos de autores como Parveen et al. (2014), quienes afirman que las redes sociales son usadas para la promoción y publicidad, el branding y, en mucha menor medida, para comunicar y dialogar sobre las actividades de RSE.

Las empresas principalmente comparten información sobre temas de negocios (y básicamente de su oferta comercial) en sus fanpages corporativas. Los temas de RSE pocas veces son tratados en las publicaciones de las empresas. Sin embargo, es necesario destacar que las empresas de América Latina los tratan con más frecuencia que las empresas globales, las cuales se centran con mayor intensidad que las primeras en los temas de negocios. Este es un dato relevante porque la comunicación de la RSE contribuye a impulsar y fortalecer la reputación corporativa, y lo que demuestra el estudio es que este aspecto clave de la gestión de la comunicación es poco considerado.

De las temáticas de RSE analizadas, tanto las empresas de América Latina como las empresas globales están enfocándose principalmente en la comunicación de los temas sociales y medioambientales, por lo que dan muy poco espacio e interés a los temas sobre gobierno corporativo y ética, y los recursos humanos y el empleo. La gestión estratégica de la comunicación de la RSE en las empresas está enfocada directamente en uno de los 
tres aspectos fundamentales del área (la ciudadanía), ya que la presencia de posts vinculados con la integridad y el trabajo es casi testimonial.

Estos resultados reafirman lo mencionado por algunos autores (Eisenegger \& Schranz, 2011; Haigh et al., 2013), quienes sostienen que los contenidos que difunden las empresas están centrados principalmente en los negocios. Tal como se afirma en el marco teórico, las redes sociales son herramientas claves para la comunicación de la de RSE (Capriotti, 2011; Cortado \& Chalmeta, 2016; Gómez, 2013) y, por tanto, deberían ser incluidas en la estrategia de comunicación de las organizaciones. No obstante, en los resultados se observa que las empresas pocas veces utilizan sus fanpages corporativas de Facebook para la comunicación de las actividades de RSE.

Los resultados también indican que las empresas de América Latina y las empresas globales están utilizando mayoritariamente recursos gráficos para la difusión de los contenidos (texto e imagen) y desaprovechando ampliamente los recursos audiovisuales que permiten contribuir efectivamente a la actividad comunicativa de la RSE (las imágenes animadas y los audio-videos). Este es un dato relevante porque los estudios demuestran que el uso de recursos audiovisuales también contribuye a incrementar el nivel de engagement (Pletikosa \& Michahelles, 2013; Quintly, 2016) e incentivar a los usuarios a generar interacciones a partir de esos contenidos compartidos por las empresas.

Así pues, las empresas de América Latina (al igual que las empresas globales) tienen una presencia destacada y una actividad bastante frecuente en Facebook, pero no tan buena como cabría de esperar en temas de RSE. La comunicación está enfocada en los temas de negocios y da poco espacio a los temas de RSE, y principalmente se están usando recursos gráficos para difundir los contenidos, a la vez que se están desaprovechando los recursos interactivos y audiovisuales que permiten que los contenidos sean presentados de forma interactiva y motiven el diálogo con los públicos.

Desde el momento en que las empresas incluyen a Facebook en sus estrategias de comunicación de RSE, comienzan a utilizar una herramien- 
ta clave para generar relaciones e interactuar con sus públicos online. Para ello, mantener una presencia y actividad de forma activa, compartir contenidos vinculados con la actividad económica y social de forma similar y equitativa, y fomentar la interacción contribuyen a lograr una gestión exitosa de la comunicación de RSE con sus públicos en la red social. Las empresas reconocen la importancia de estar presentes de manera activa y permanente en Facebook, pero tienen un enfoque de comunicación oneway. La comunicación unidireccional y el escaso interés en interactuar con los públicos puede generar descontento y como consecuencia afectar negativamente la reputación corporativa online. Las empresas están utilizando Facebook como un canal de difusión más que como un canal de comunicación de su RSE. A pesar de su nivel destacado de presencia y actividad, no están gestionando la comunicación con los públicos en Facebook para establecer relaciones, sino principalmente para tener visibilidad, por lo que desaprovechan las oportunidades que le brinda la red social para la comunicación de las actividades de RSE, que contribuyen positivamente a la reputación corporativa.

En este sentido, las empresas deberían modificar el enfoque de comunicación en Facebook e integrar adecuadamente los aspectos sociales a sus estrategias de contenido en esta red. Asimismo, la atención no debería estar orientada a la difusión de información, sino más bien a contribuir y facilitar el diágolo y la interacción con los públicos en relación con los temas de RSE e integrarlos en sus actividades de RSE.

\section{Referencias}

Abitbol, A. \& Lee, S. Y. (2017). Messages on CSR-dedicated Facebook pages: What works and what doesn't. Public Relations Review, 43(4), 796-808. http://doi.org/10.1016/j.pubrev.2017.05.002

Alburquerque Llorens, F. (2004). Desarrollo económico local y descentralización en América Latina. Revista de la Cepal, 82, 157-171. Recuperado de http://observ-ocd.org/sites/observ-ocd.org/files/ publicacion/docs/350_177.pdf 
Capriotti, P. (2011). Communicating corporate social responsibility through the internet and social media. En Ø. Ihlen, J. L. Bartlett \& S. May (Eds.), The handbook of communication and corporate social responsibility (pp. 358-378). Boston, EE. UU.: Wiley-Blackwell. http://doi.org/10.1002/9781118083246

Capriotti, P. (2017). The world wide web and the social media as tools of CSR communication. En S. Diehl, M. Karmasin, B. Mueller, R. Terlutter \& F. Weder (Eds.), Handbook of integrated CSR communication (pp. 193-2010). Cham, Alemania: Springer. http://doi. org/10.1007/978-3-319-44700-1

Capriotti, P., Carretón, C. \& Castillo, A. (2016). Testing the level of interactivity of institutional websites: From museums 1.0 to museums 2.0. International Journal of Information Management, 36(1), 97104. http://doi.org/10.1016/j.ijinfomgt.2015.10.003

Capriotti, P. \& Moreno, Á. (2007). Corporate citizenship and public relations: The importance and interactivity of social responsibility issues on corporate websites. Public Relations Review, 33(1), 84-91. http://doi.org/10.1016/j.pubrev.2006.11.012

Capriotti, P. \& Pardo Kuklinski, H. P. (2012). Assessing dialogic communication through the internet in Spanish museums. Public Relations Review, 38(4), 619-626. http://doi.org/10.1016/j.pubrev.2012.05.005

Carroll, A. B. (1999). Corporate social responsibility. Business and Society, 38(3), 268-295. https:/doi.org/10.1177/000765039903800303

Carroll, A. B. (2016). Carroll's pyramid of CSR: taking another look. International Journal of Corporate Social Responsibility, 1(1). https:// doi.org/10.1186/s40991-016-0004-6

Cho, M., Furey, L. D. \& Mohr, T. (2016). Communicating corporate social responsibility on social media: Strategies, stakeholders, and 
public engagement on corporate Facebook. Business and Professional Communication Quarterly, 80(1), 52-69. http://doi. org/10.1177/2329490616663708

Cortado, F.J. \& Chalmeta, R. (2016). Use of social networks as a CSR communication tool. Cogent Business \& Management, 3(1), 1187783. https://doi.org/10.1080/23311975.2016.1187783

Devaney, E. (2015). Social media benchmarks report 2015. Recuperado de https://cdn2.hubspot.net/hub/53/file-2415418647-pdf/00OFFERS-HIDDEN/social-media-benchmarks-2015. pdf?t=1423113374840

Dijkmans, C., Kerkhof, P. \& Beukeboom, C. J. (2015). A stage to engage: Social media use and corporate reputation. Tourism Management, 47, 58-67. https://doi.org/10.1016/j.tourman.2014.09.005

Eisenegger, M. \& Schranz, M. (2011). Reputation management and corporate social responsibility. En Ø. Ihlen, J. L. Bartlett \& S. May (Eds.), The handbook of communication and corporate social responsibility (pp. 129-146). Sussex, RU: Wiley-Blackwell.

Esrock, S. L. \& Leichty, G. B. (1998). Social responsibility and corporate web pages: Self-presentation or agenda-setting? Public Relations Review, 24(3), 305-319. https://doi.org/10.1016/ S0363-8111(99)80142-8

Estudio de Comunicación. (2017). Presencia de las empresas del Ibex35 en el entorno digital. Recuperado de https://www.estudiodecomunicacion.com/servicios/presencia-de-las-empresas-del-ibex35-enel-entorno-digital/

Fosk, A. (2015, mayo 22). Futuro Digital América Latina 2015. Recuperado de http://www.comscore.com/lat/FuturoDigital2015 
Gómez Vásquez, L. (2013). Me gusta o te sigo: análisis de la comunicación de prácticas de responsabilidad social corporativa a través de los medios sociales. Correspondencias \& Análisis, 3, 89-109. Recuperado de https://dialnet.unirioja.es/servlet/articulo? codigo $=4739318$

Guillory, J. E. \& Sundar, S. S. (2014). How does web site interactivity affect our perceptions of an organization? Journal of Public Relations Research, 26(1), 44-61. https://doi.org/10.1080/106272 6X.2013.795866

Haigh, M. M., Brubaker, P. \& Whiteside, E. (2013). Facebook: Examining the information presented and its impact on stakeholders. Corporate Communications: An International Journal, 18(1), 52-69. https:// doi.org/10.1108/13563281311294128

Invodo. (2016). Video Statistics: The Marketer's Summary 2016. Recuperado de http://www.webvideomarketing.org/pdf/Video-StatisticsThe-Marketers-Summary-2016-invodo.pdf

Jordan, R. (2017, junio 24). How Frequently Should I Post to Facebook? Recuperado de https://www.929marketing.com/blog/how-frequentlyshould-i-post-to-facebook

Kemp, S. (2017, enero 24). Digital in 2017: Global Overview. Recuperado de https://wearesocial.com/special-reports/digital-in-2017-global-overview

Ki, E.-J. \& Nekmat, E. (2014). Situational crisis communication and interactivity: Usage and effectiveness of Facebook for crisis management by Fortune 500 companies. Computers in Human Behavior, 35, 140-147. https://doi.org/10.1016/j.chb.2014.02.039

Kim, S. (2019). The process model of corporate social responsibility (CSR) communication: CSR communication and its relationship with consumers' CSR knowledge, trust, and corporate reputation per- 
ception. Journal of Business Ethics, 154(4), 1143-1159. https:// doi. org/10.1007/s10551-017-3433-6

Kim, S., Kim, S.-Y. \& Hoon Sung, K. (2014). Fortune 100 companies' Facebook strategies: Corporate ability versus social responsibility. Journal of Communication Management, 18(4), 343-362. https:// doi.org/10.1108/JCOM-01-2012-0006

Losada-Díaz, J. C. y Capriotti, P. (2015). La comunicación de los museos de arte en Facebook: comparación entre las principales instituciones internacionales y españolas. Palabra Clave, 18(3), 889-904. https://doi.org/10.5294/pacla.2015.18.3.11

Luarn, P., Lin, Y.-F. \& Chiu, Y.-P. (2015). Influence of Facebook brand-page posts on online engagement. Online Information Review, 39(4), 505-519. https://doi.org/10.1108/OIR-01-2015-0029

Masoud, N. (2017). How to win the battle of ideas in corporate social responsibility: The International Pyramid Model of CSR. International Journal of Corporate Social Responsibility, 2(1). https://doi. org/10.1186/s40991-017-0015-y

Moreno, A., Molleda, J. C., Athaydes, A., Suárez, A. M., Herrera, M. \& Álvarez, A. (2017). Latin American Communication Monitor 2016-2017. Tendencias en comunicación estratégica: big data, automatización, engagement, influencers, coaching y competencias. Resultados de una encuesta en 17 países. Madrid, España: EUPRERA/DIRCOM.

Navarro, C., Moreno, Á. \& Al-Sumait, F. (2017). Social media expectations between public relations professionals and their stakeholders: Results of the ComGap study in Spain. Public Relations Review, 43(4), 700-708. https://doi.org/10.1016/j.pubrev.2016.12.008

Parveen, F., Jaafar, N. I. \& Ainin, S. (2014). Social media usage and organizational performance: Reflections of Malaysian social media 
managers. Telematics and Informatics, 32(1), 67-78. https://doi. org/10.1016/j.tele.2014.03.001

Patel, N. (2016, septiembre 12). How frequently you should post on social media according to the pros. Recuperado de https://www.forbes.com/ sites/neilpatel/2016/09/12/how-frequently-you-should-post-onsocial-media-according-to-the-pros/-4356de7e240f

Peinado-Vara, E. (2011). RSE en América Latina. En A. Vives y E. Peinado-Vara (Eds.), La responsabilidad social de la empresa en América Latina (pp. 65-83). Nueva York, EE. UU. Banco Interamericano de Desarrollo. Recuperado de http://www.cepb.org.bo/urse/wpcontent $/$ uploads $/ 2014 / 12 /$ E21.pdf-page $=67$

Pletikosa Cvijikj, I. \& Michahelles, F. (2013). Online engagement factors on Facebook brand pages. Social Network Analysis and Mining, 3(4), 843-861. https://doi.org/10.1007/s13278-013-0098-8

Leoprechting, L. von. (2016, octubre 19). 30 biggest brands on Facebook analyzed in-depth. Recuperado de https://www.quintly.com/ blog/2016/10/30-biggest-brands-on-facebook-analyzed-indepth/

Taylor, M. \& Kent, M. L. (2014). Dialogic engagement: Clarifying foundational concepts. Journal of Public Relations Research, 26(5), 384398. https://doi.org/10.1080/1062726X.2014.956106

Ure, M. (2016). La comunicación de la administración pública en las redes sociales: los casos de las ciudades de Buenos Aires y Bolonia. Palabra Clave, 19(1), 240-270. https://doi.org/10.5294/ pacla.2016.19.1.10

Waddock, S. (2004). Parallel universes: Companies, academics, and the progress of corporate citizenship. Business and Society Review, 109(1), 5-42. https://doi.org/10.1111/j.0045-3609.2004.00002.x

26 Comunicación de la responsabilidad social empresarial de las empresas de América... - Paul Capriotti y otra 
Waters, R. D., Burnett, E., Lamm, A. \& Lucas, J. (2009). Engaging stakeholders through social networking: How nonprofit organizations are using Facebook. Public Relations Review, 35(2), 102-106. https:// doi.org/10.1016/j.pubrev.2009.01.006

Wood, D. J. (1991). Corporate social performance revisited. Academy of Management Review, 16(4), 691-718. https://doi. org/10.2307/258977

Yudira, Z. (2007). La inversión extranjera directa de la unión europea hacia américa latina: el método de entrada de las principales multinacionales eléctricas. Estudios Gerenciales, 23(105), 39-55. https:// doi.org/10.1016/S0123-5923(07)70024-4 


\section{Anexo 1. Ficha técnica}

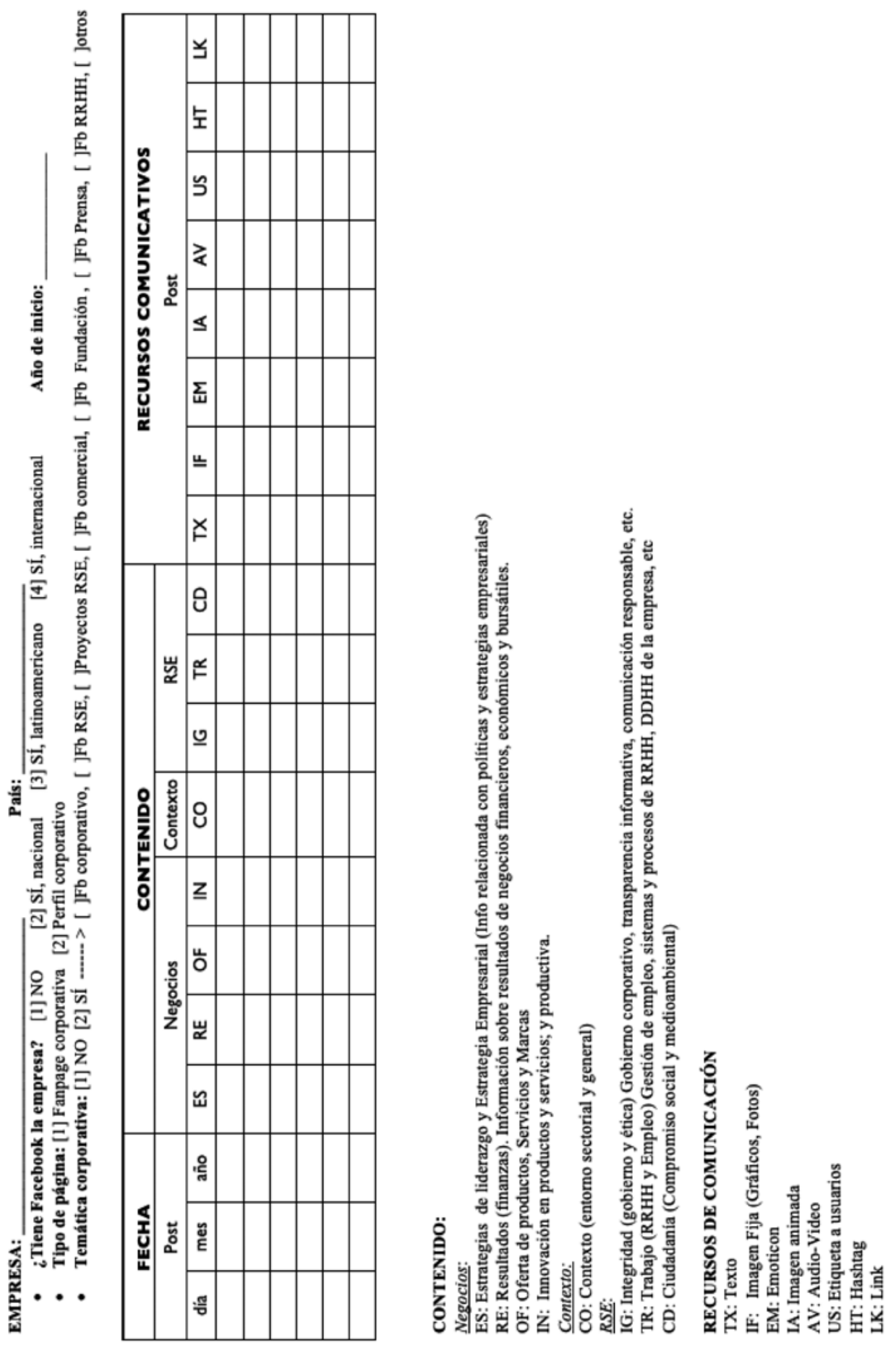

